\title{
Virtual Reality for Training Doctors to Break Bad News
}

\author{
Magalie Ochs ${ }^{1}$ and Philippe Blache ${ }^{2}$ \\ Aix Marseille Université, CNRS, ENSAM, Université de Toulon, 13397 Marseille, France \\ ${ }^{1}$ Laboratoire des Sciences de l'Information et des Systèmes, LSIS, UMR7296 \\ magalie.ochs@lsis.org \\ ${ }^{2}$ Laboratoire Parole et Langage, LPL, UMR7309 \\ philippe.blacheelpl.fr
}

\begin{abstract}
The way doctors deliver bad news has a significant impact on the therapeutic process: disease evolution, adherence with treatment recommendations, litigation possibilities (Andrade et al., 2010). However, both experienced clinicians and medical trainees consider this task as difficult, daunting, and stressful. Nowadays, training health care professional to break bad news, recommended by the French Haute Autorité de la Santé (HAS), is organized as workshops during which doctors disclose bad news to actors playing the role of patient. In our project, we are developing an embodied conversational agent simulating a patient to train doctors to break bad news. The embodied conversational agent is incorporated in an immersive virtual reality environment (a CAVE) integrating several sensors to detect and recognize in real time the verbal and non-verbal behavior of the doctors interacting with the virtual patient. The virtual patient will adapt its behavior depending on the doctor's verbal and non-verbal behavior. The methodology used to construct the virtual patient behavior model is based on a quantitative and qualitative analysis of corpus of doctors training sessions.
\end{abstract}

Keywords: Embodied conversational agent, virtual reality, virtual patient, training platform.

\section{Introduction}

The way doctors deliver bad news has a significant impact on the therapeutic process: disease evolution, adherence with treatment recommendations, litigation possibilities (Andrade et al., 2010). However, both experienced clinicians and medical trainees consider this task as difficult, daunting, and stressful. Nowadays, training health care professional to break bad news, recommended by the French Haute Autorité de la Santé (HAS), is organized as workshops during which doctors disclose bad news to actors playing the role of patient. This training solution requires a huge amount of human resources as well as high level of preparation (each $30 \mathrm{mn}$ session requires an hour of preparation), not to speak about funding. It is then difficult to propose the

adfa, p. 1, 2011.

(C) Springer-Verlag Berlin Heidelberg 2011 
student a large variety of situation or patient profiles. It is then necessary to make use of information technologies applied to learning.

In this project, we aim at developing an embodied conversational agent (ECA) simulating a patient. Such a platform would play a decisive role for institutions involved in training (hospitals, universities): the needs concern potentially thousands of doctors/students. Organizing such training at this scale is not realistic with human actors. A virtual solution would be then an adequate answer.

Our objective is to develop an immersive platform that enables doctors to train to break bad news with a virtual patient. For this purpose, we adopt a multidisciplinary approach in the project gathering computer scientists, linguists, psychologists and doctors. The objective of the paper is to present the global project (motivation, scientific challenges, and methodology). In the following, after a presentation of a state of art in this domain (Section 2), we present the corpus-based approach used to model the virtual patient (Section 3) and we introduce the training platform and its different components (Section 4).

\section{State of Art}

For several years, there has been a growing interest in Embodied Conversational Agents (ECAs) to be used as a new type of human-machine interface. ECAs are autonomous entities, able to communicate verbally and nonverbally. They can attract and maintain the attention of users in an interaction, to make the interaction more expressive and more socially adapted. Indeed, several researches have shown that embodied conversational agents are perceived as social entities leading users to show behaviors that would be expected in human-human interactions (Krämer, 2005).

Moreover, recent research showed that virtual agents could help human beings improve their social skills. For instance, in (Finkelstein et al., 2013), a virtual agent is used to train kids to adapt their language register to the situation. In the European project TARDIS (Anderson et al., 2013), an ECA endowed the role of a virtual recruiter is used to train young adults to job interview. The E-circus project offers children a role playing environment with expressive virtual agents to learn how to deal with bullying situations at school (Weiss et al., 2007). This research shows that embodied conversational agent can be used for social training since users will react to the ECA in a similar way that to another person and the socio-emotional responses of the agents will help them practice and improve their social skills.

Several ECAs embodied the role of virtual patients have already been proposed for use in clinical assessments, interviewing and diagnosis training (Cavazza and Simo, 2003; Andrade et al., 2011; Johnsen et al., 2005; Kenny et al., 2008; Lock et al., 2006). Indeed, previous research has shown that doctors demonstrate non-verbal behaviors and respond empathetically to a virtual patient (Deladisma et al., 2006). In this domain, the research has mainly focused on the anatomical and physiological models of the virtual patient to simulate the effects of medical interventions or on models to simulate particular disorder. For instance, Justina is a virtual patient simulating Post Traumatic Stress Disorder (PTSD) to train medical students' interview 
skills and diagnostic acumen for patient with such disorder (Kenny et al., 2008). DIANA (DIgital ANimated Avatar) is a female virtual character playing the role of a patient with appendicitis (Lock et al., 2006). In the eViP European project (http://www.virtualpatients.eu), the objective is specifically to develop a large amount of virtual patients simulating different pathologies. In our project, we focus on a virtual patient to train doctors to deliver bad news.

A first study (Andrade et al., 2010) has analyzed the benefits of using a virtual patient to train doctors to deliver bad news. The results show significant improvements of the self-efficacy of the medical trainees. The participants consider the virtual patient as "excellent instructional method for learning how to deliver bad news". The major limit of the proposed system, highlighted by the participants, is the lack of nonverbal behaviors of the patients simulated in the limited environment Second Life (Linden Labs, San Francisco, CA). Our objective in this project is to simulate the nonverbal emotional expression of the virtual patient to improve the believability of the virtual character and the immersive experience of the doctor.

Most of the embodied conversational agents used for health applications have been integrated in 3D virtual environment on PC. Virtual reality in health domain is particularly used for virtual reality exposure therapy (VRET) for the treatment for anxiety and specific phobias. For instance, people with a fear of public speaking may speak to an audience of virtual characters in virtual reality environment to reduce their anxiety in reality (Parsons and Rizzo, 2008). In our project, in order to offer an immersive experience to the doctor, we have integrated the virtual patient in a virtual reality environment.

Several researches have been conducted to simulate emotional state through the non-verbal behavior of embodied conversational agent. However, expression of emotions in virtual agents (e.g. Pandzic \& Forcheimer 2002) often relies on the six prototypical expressions of emotions (joy, fear, anger, disgust, sadness, and surprise). Moreover, in the context of breaking bad news, the virtual agent has to be able to display specific panel of strong emotions and feelings (such as tears) through particular speech, postures, gestures and facial expressions. To our knowledge, no existing computational model has been developed to simulate the effect of strong negative emotions on the behavior (verbal and non-verbal) of a virtual character.

Moreover, a virtual patient should be able to display verbal and non-verbal reactions appropriately according to the doctor's behavior. During an interaction, the interlocutors in fact coordinate or align their verbal and non-verbal behavior (e.g. feedback, mimicry). According to the Communication Accommodation theory (CAT), the interlocutors adapt the coordination of their behavior to express different social attitudes (Gilis et al., 1991). Recent research in human-machine interaction confirms this hypothesis: the coordination of the virtual agent's behavior on the one of the user or the divergence of its behavior reflects the agent's social attitude (e.g. appreciation, cold, mutual understanding, etc.) (e.g. Bailenson, et al., 2005). In the project described in this paper, sensors will be used to automatically detect in real-time the verbal and non-verbal behavior of the doctors during their interaction with the virtual patient. These inputs will then be used by the virtual patient to coordinate its behavior on the doctor's one depending on the social attitude to express. The methodology 
used to define the virtual agent's behavior is based on the analysis of a corpus of doctor-patient interaction to extract rules on its verbal and non-verbal reactions.

\section{A corpus-based approach to simulate a virtual patient}

For ethical reasons, it is not possible to videotape real breaking bad news situations. Instead, simulations are organized with actors playing the role of the patient. A corpus

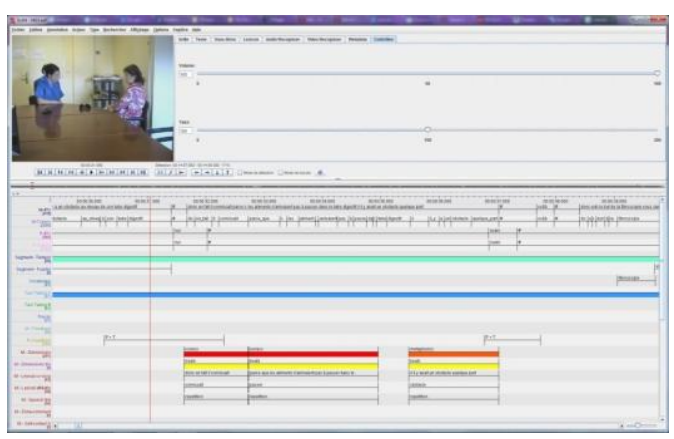

Fig. 1: Corpus annotation with Elan of such interactions has been collected in different medical institutions (the Institut Paoli Calmette and the hospital of Angers). Simulated patients are actors trained to play the most frequently observed patients reactions (denial, shock...). The actor follows a pre-determined scenario. The doctor (i.e. the trainee) receives details of a medical case before the simulated interaction starts (patient medical history, family background, surgery, diagnosis, etc.). On average, a simulated consultation lasts 30 minutes. The collected corpus is currently composed of 23 videos of patientdoctor interaction with different scenario (e.g. patient aggressive or accommodating).

These simulated interactions of the collected corpus are transcribed and annotated (with Elan software - Figure 1) on several levels: at the discourse level (e.g. dialog phases, turn-taking) and at non-verbal levels (e.g. feedback, gaze, gestures, etc.). The coding scheme has been defined based on a preliminary analysis of the corpus (Saubesty and Tellier, 2015). The objective of the annotation is to highlight the necessary information to model and implement a virtual patient and reproduce this kind of interaction.

The method used to extract information from the annotated corpus is based on a multidisciplinary approach combining (1) a manually analysis of the data by linguists for a qualitative analysis and (2) automatic requests (using the SPPAS software (Bigi, 2015)) and datamining algorithms (e.g. (Rabatel et al., 2010) on the data for a more quantitative study performed by computer scientists.

\section{$4 \quad$ Virtual reality platform of training}

The tool we use to animate the virtual patient is the Greta System (Pelachaud, 2009). Greta offers several modules, each dedicated to particular functionality to both design new facial expressions and gestures and to animate in real-time 3D virtual agents in virtual environments. The lexicon of gestures of Greta is enriched by specific gestures and facial expressions of patients identified in the corpus. 
The virtual patient has been integrated in the CRVM (Centre de Réalité Virtuelle de

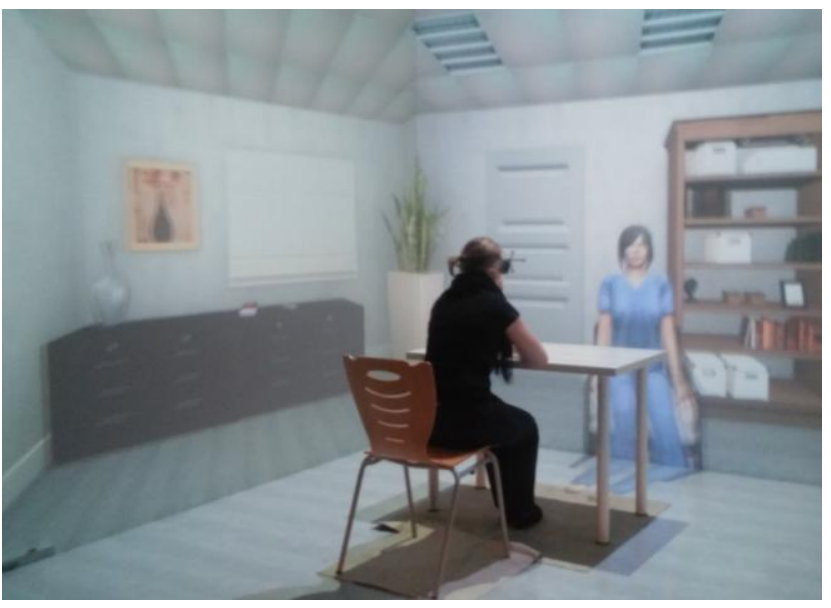

Fig. 2: Virtual reality environment for training
Marseille, platform of the ISM partner). The visualization system consists of a high-end platform called "CAVE TM". CAVE TM is composed of four projection screens: frontal, ground and lateral projections. Each frontal and lateral screen has a projection surface of 3 meters wide by 4 meters high (Figure 2). The speech of the doctor is recognized by an automatic speech recognition system

(Nocera et al., 2002). We are improving the system by learning the lexicon and language model of the speech recognition system on the transcript corpus of patientdoctor interactions. For this project, we have defined a specific use case that reflects a real situation in which doctors may train their social competences in delivering bad news. It is based on real scenario of training including some possible variations (e.g. patient aggressive or accommodating). The dialog model of the patient is based on this scenario. We are defining the dialog rules that depend on the profile of the patient (aggressive or accommodating) and the discourse of the trainee (e.g. level of details, use of medical terms, etc.). The dialog rules and more generally the behavior of the virtual patient are based on the corpus analysis (as described in the previous section).

To consider the non-verbal behavior of the trainee, on the table in the CAVE, we will install a Kinect to automatically detect some non-verbal signals of the doctors (e.g. the gaze direction, head movements, posture, and facial expressions). The objective is to coordinate or in contrary discoordinate the non-verbal behavior of the virtual patient on those of the trainee depending on the attitude that the virtual patient should convey.

\section{References}

1. K. Anderson, E. André, T. Baur, S. Bernardini, M. Chollet, E. Chryssafidou, I. Damian, C. Ennis, A. Egges, P. Gebhard, H. Jones, M. Ochs, C. Pelachaud, K. Porayska-Pomsta, P. Rizzo, and N. Sabouret The TARDIS Framework: Intelligent Virtual Agents for Social Coaching in Job Interviews Advances in Computer Entertainment (ACE'13).

2. Andrade AD, Bagri A, Zaw K, Roos BA, Ruiz JG. Avatar-mediated training in the delivery of bad news in a virtual world. J Palliat Med 2010;13:1415-9. 
3. Bailenson, J.N., Swinth, K. R., Hoyt, C. L., Persky, S., Dimov, A., and Blascovich, J. (2005). The independent and interactive effects of embodied agent appearance and behavior on self-report, cognitive, and behavioral markers of copresence in immersive virtual environments. Presence: Teleoperators and Virtual Environments, 14, 379-393.

4. Bigi, B. (2015). SPPAS - Multi-lingual Approaches to the Automatic Annotation of Speech the Phonetician - International Society of Phonetic Sciences, ISSN 0741-6164, Number 111-112 / 2015-I-II, pages 54-69.

5. Cavazza and A. Simo. 2003. A virtual patient based on qualitative simulation. In Proceedings of the 8th international conference on Intelligent user interfaces (IUI '03). ACM, New York, NY, USA, 19-25.

6. A. M. Deladisma, M. Cohen, A. Stevens et al., Do medical students respond empathetically to a virtual patient?, Association for Surgical Education meeting, 2006.

7. Finkelstein, S., Yarzebinski, E., Vaughn, C., Ogan, A., \& Cassell, J. (2013) "The effects of culturally-congruent educational technologies on student achievement". in Proceedings of Artificial Intelligence in Education (AIED)

8. H. Giles, N. Coupland, \& J. Coupland (1991). Accommodation Theory: Communication, Context and Consequence. Journal of Context of Accommodation: Development in Applied Sociolinguistics, p.27Gratch et al., 2006

9. K. Johnsen, R. Dickerson, A. Raij, B. Lok, J. Jackson, M. Shin, J. Hernandez, A. Stevens, and D. Scott Lind. 2005. Experiences in Using Immersive Virtual Characters to Educate Medical Communication Skills. In Proceedings of the 2005 IEEE Conference 2005 on Virtual Reality (VR '05). IEEE Computer Society, Washington, DC, USA, 179-186, 324.

10. P. Kenny, T. D. Parsons, J. Gratch, and A. A. Rizzo. 2008. Evaluation of Justina: A Virtual Patient with PTSD. In Proceedings of the 8th international conference on Intelligent Virtual Agents (IVA '08), Helmut Prendinger, James Lester, and Mitsuru Ishizuka (Eds.). Springer-Verlag, Berlin, Heidelberg, 394-408.

11. Krämer N., Iurgel I., Bente G., (2005). Emotion and motivation in embodied conversational agents. Canamero, L. (ed.), Proceedings of the Symposium 'Agents that Want and Like', AISB 2005, 55-61. Hatfield: SSAISB

12. B. Lok, R. E. Ferdig, A. Raij, K. Johnsen, R. Dickerson, J. Coutts, A. Stevens, and D. S. Lind. 2006. Applying virtual reality in medical communication education: current findings and potential teaching and learning benefits of immersive virtual patients. Virtual Real. 10,

13. Nocera, P., Linares, G., Massonié, D., and Lefort, L. (2002) "Phoneme lattice based a* search algorithm for speech recognition" in International Conference on Text, Speech and Dialogue, pages 301-308, Brno, Springer

14. I.S. Pandzic, and R. Forchheimer, editors (2002). MPEG-4 Facial Animation - The standard, implementations and applications. John Wiley \& Sons, 2002.

15. T.D. Parsons, A.A. Rizzo (2008). "Affective outcomes of virtual reality exposure therapy for anxiety and specific phobias: A meta-analysis". Journal of Behavior Therapy and Experimental Psychiatry 39 (3): 250-261.

16. C. Pelachaud, Studies on Gesture Expressivity for a Virtual Agent, Speech Communication, special issue in honor of Björn Granstrom and Rolf Carlson, 51 (2009) 630-639

17. J. Rabatel, S. Bringay and P. Poncelet. 2010. Contextual Sequential Pattern Mining. IEEE International Conference on Data Mining Workshops. December, 2010 , pp. 981-988

18. Saubesty, J. \& Tellier, M. (2015) Multimodal analysis of hand gesture back-channel feedback. Gesture and Speech in Interaction, Sept. 2015 Nantes, France.

19. Weiss, L. C. Oliveira, S. Paulo, C. Mendes, L. Figueira, Marco Vala, P. Sequeira, A. Paiva, T. Vogt, E. Andre, "ECIRCUS: Building Voices for Autonomous Speaking Agents", 6th Speech Synthesis Workshop, Aug. 2007, ISCA. 